\title{
Secondary reinforcement strength with continuous primary reinforcement: Fixed-ratio and continuous secondary reinforcement schedules
}

\author{
MATTHEW J. SWIERGOSZ and HARVARD L. ARMUS \\ University of Toledo, Toledo, Ohio
}

\begin{abstract}
Three groups of rats were trained on a leverpressing task in which all groups received continuous food reinforcement. Two of the groups were trained with an equal number of leverpresses, one with continuous secondary reinforcement and the other with secondary reinforcement on a fixed-ratio schedule. A third group was given additional training trials on a fixed ratio of secondary reinforcement. The subjects were tested using a two-lever choice technique without primary reinforcement. Pressing one lever produced a light-sound stimulus that had previously served as the secondary reinforcing stimulus, whereas pressing the other lever did not. The primary finding revealed no significant differences in secondary reinforcement strength between the continuous reinforcement and the fixed-ratio-4 secondary reinforcement groups, supporting previous results.
\end{abstract}

In 1962, Egger and Miller proposed that, to become an effective secondary reinforcer, a stimulus must be a reliable and nonredundant predictor of the occurrence of primary reinforcement. Comparisons between continuous reinforcement (CRF) and partial reinforcement (PR) schedules of primary reinforcement showed that secondary reinforcement was more effective in the PR group, provided the secondary reinforcing stimulus was more predictive of primary reinforcement for this group than for the CRF group (Armus, DeVoy, Eisenberg, \& Schroeder, 1962; Armus \& Garlich, 1961). In contrast, when subjects were trained on a combined CRF/PR schedule, with each subject receiving blocks of trials under each schedule, the CRF-associated stimulus acquired greater secondary reinforcement strength (Armus et al., 1964). However, the studies of Armus and Garlich (1961) and Armus et al. (1962) lacked a control for the schedule of presentation of the secondary reinforcement stimulus $\left(\mathrm{S}^{\mathrm{r}}\right)$. The potential $S^{\mathrm{r}}$ was presented on a fixed-ratio (FR) schedule for the FR food-reinforced group, and on a CRF schedule for the CRF food-reinforced group. The results of these studies might have been a function of the different schedules of $S^{r}$ presentation rather than of the differential predictiveness of food reinforcement.

The present study was designed to assess this possibility by investigating the effects of the schedule of $S^{\mathrm{T}}$ presentation (i.e., continuous or fixed-ratio schedules). A secondary purpose was to examine the effect of varying the number of primary-secondary reinforcement pairings during the acquisition of secondary reinforcement.

Address correspondence to Harvard L. Armus, Department of Psychology, University of Toledo, $2801 \mathrm{~W}$. Bancroft St., Toledo, OH 43606 .

\section{METHOD}

\section{Subjects and Apparatus}

The subjects were 30 naive, male Long-Evans rats, approximately 90 or 105 days old at the beginning of training. They were randomly divided into three groups. Three rats were eliminated because of illness and apparatus failure.

The apparatus was a $20 \times 11 \times 14 \mathrm{~cm}$ operant chamber with transparent Plexiglas side walls, aluminum front and back walls, and a brass rod floor. Depending upon the phase of the experiment, the box was fitted with one retractable center lever or two retractable side levers, with a minimum lever-operating force of 8-9 g. A small 3-W lamp with a white, faceted jewel was mounted above and below each lever. Following a response, the lever(s) were withdrawn from the box for 5-6 sec by a motor-driven unit. The lever(s) were electrically inactive during this interval. Reinforcement was one 45-mg Noyes food pellet. The box was dimly illuminated by a small 7-W orange lamp and mounted inside a ventilated refrigerator shell. Observation was possible through a double one-way window incorporated into the refrigerator shell. The temperature was maintained at approximately $71^{\circ} \mathrm{F}$.

\section{Procedure}

Approximately 2 weeks before training, the subjects were weighed and placed on a feeding schedule to stabilize the weight of each subject at $80 \%$ of his predeprivation weight.

The three groups were trained on the leverpress response with continuous food reinforcement, but training differed in terms of the percentage of trials on which food reinforcement was accompanied by the light-sound stimulus that was to function as the potential $S^{r}$. Group CRF/CRF received the $S^{r}$ with each leverpress; Group CRF/FR-4 received the $S^{r}$ with every fourth leverpress; and Group CRF/FR-4x was identical to Group CRF/FR-4, except that the animals made four times as many leverpresses and received four times as many $S^{\mathrm{T}}$ presentations. In each case, the designation before the slash refers to the schedule of primary reinforcement (always CRF), and the designation following the slash refers to the schedule of $S^{r}$ presentation.

Over 10 training days, Groups CRF/CRF and CRF/FR-4 completed approximately 400 trials, and Group CRF/FR-4x completed 1,600 trials. During training, reinforced trials were accompanied by the noise of the pellet dispenser and a 2 -sec flash of both center-lever lights, according to the respective schedules. This stimulus combination was the potential $S^{r}$. 


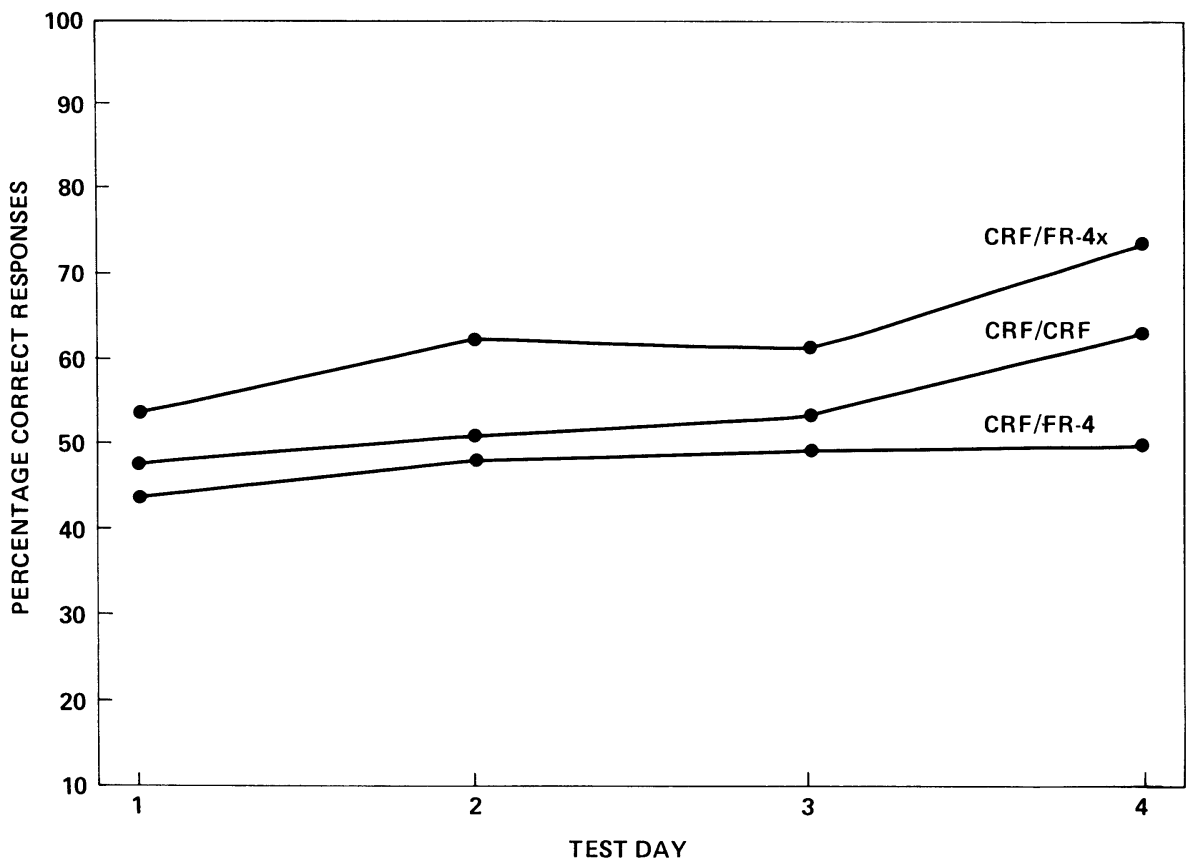

Figure 1. Percentage of secondarily reinforced (correct) responses for each of the 4 test days.

Secondary reinforcement testing took place on the day immediately following the last training day. There were 4 test days, each with a maximum of 50 responses. The two side levers were substituted for the center lever in this phase. No food reinforcement was given; the pellet dispenser was emptied, and the pellet chute was disconnected from the apparatus. Each group was randomly divided, so that the right lever was correct for half of the subjects in each group, and the left lever was correct for the other half. A correct response produced the 2-sec flash of the center-lever lights accompanied by the noise of the empty pellet dispenser, and an incorrect response did not. After each response, both levers were withdrawn from the box for 5-6 sec.

\section{RESULTS AND DISCUSSION}

Figure 1 shows the percentages of correct responses, that is, responses producing the $S^{r}$ during the 4 test days. Analysis of variance revealed a significant effect for $\mathrm{S}^{\mathrm{r}}$ presentation condition $[F(2,24)=4.04, p<.05]$ and for test days $[F(3,72)=3.66, p<.05]$, but no significant interaction. The $t$ tests showed no significant difference between Group CRF/CRF and the other two groups. However, Group CRF/FR-4x had a greater percentage of correct responses than Group CRF/FR-4 $[t(16)=2.77$, $p<.05]$. The $t$ tests further showed that there were no significant differences between any of the groups on
Trial 1 of Test Day 1, indicating a lack of initial group bias.

Because no significant secondary reinforcement strength differences were found between Group CRF/CRF and the other two groups, the schedule of $S^{r}$ presentation was not a factor in determining secondary reinforcement strength.

\section{REFERENCES}

Armus, H. L., DeVoy, W. E., Eisenberg, T., \& Schroeder, S. R. (1962). Effect of primary reinforcement schedule on secondary reinforcement strength with continuous secondary reinforcement during training. Psychological Reports, 11, 203-208.

ARMUS, H. L., \& GARLICH, M. M. (1961). Secondary reinforcement strength as a function of schedule of primary reinforcement. Journal of Comparative \& Physiological Psychology, 54, 56-58.

Armus, H. L., Guinan, J. F., Crowell, R. A., Schroeder, S. R., RudGe, J. A., \& CoмtoIs, D. R. (1964). Secondary reinforcement strength and primary reinforcement schedule: Single group technique. Journal of Comparative \& Physiological Psychology, 57, 313-315. EGGER, M. D., \& MiLLER, N. E. (1962). Secondary reinforcement in rats as a function of information value and reliability of the stimulus. Journal of Experimental Psychology, 64, 97-104.

(Manuscript received for publication October 5, 1987.) 